\title{
Digital Up and Down Converter for High Performance VHF and UHF transceiver
}

\author{
Pavel Kovář, Pavel Puričer, Tomáš Morong, Filip Šturc \\ Department of Radio Engineering \\ Czech technical university in Prague, EEL \\ Prague, Czech Republic \\ kovar@fel.cvut.cz
}

\begin{abstract}
Abstract - The Digital Up and Down Converter (DUC/DDC) is a basic building part of the professional digital mobile transceiver. The paper presents DDC/DDS for dual mode professional $\mathrm{VHF} / \mathrm{UHF}$ transceiver developed for railway radio. The transceiver complies ETSI standards for NFM and Digital Mobile Radio (DMR). The DUC/DDC is featured with the channel selectivity exceeding $70 \mathrm{~dB}$ and dynamic range more than $90 \mathrm{~dB}$. The implementation of the other standards that use constant envelope modulation like small satellite UHF communication links is also possible.
\end{abstract}

Keywords-Digital Mobile Radio; Digital Down Converter; Digital Up Converter; satellite communication

\section{INTRODUCTION}

This paper presents an architecture and performance test of the Digital Up Converter (DUC) and Digital Down Converter (DDC) for professional VHF and UHF transceiver that uses analog and digital constant envelope modulations. The DUC/DDC was originally designed in the frame of the project for innovation of professional dual mode VHF and UHF transceiver for railway with our industrial partner $\mathrm{T}$ $\mathrm{CZ}$. The design complies with the ETSI standard for analog NFM communication [1] and digital data communication [2] namely Digital Mobile Radio Standard (DMR) [3] but its application is not limited to these two standards. The other application can be seen in professional long-range digital communication with drones and UAV and communication with small satellites and other standards for professional digital mobile radio like $\mathrm{dPMR}$.

Let us note that the digital standards bring to the communication new quality $[4,5,6]$ like lower distortion, higher sensitivity and related longer range. Very important is communication privacy, encryption, and last but not least data transfer.

\section{TECHNICAL REQUIREMENTS}

ETSI standards $[1,2]$ define technical requirements on the transmitted signal like modulation, transmitter spectral mask, frequency stability, etc. The technical analysis identified the most critical parameter, which is the fulfillment of the spectral mask. The spectral mask of the transmitter is mainly affected by a quality of the modulator, (which can be close to nearly optimal performance in the digital implementation) and by SSB spectral noise of the carrier frequency signal.
The problem of the DUC converter is a complicated generation of this signal. The signal is firstly generated in digital circuits then passes through digital to analog conversion that introduces quantization noise as well as phase jitter of the sampling signal. The intermediated signal is then usually upconverted to the carrier frequency. The problem is self-mixing with the local oscillator signal that transforms the phase noise from the local oscillator to the modulated signal.

Concerning the receiver, the professional digital mobile radio standards define very tight requirements on to the channel selectivity, dynamic range, spurious response, and intermodulation suppression. The adjacent channel, as well as spurious receptions, shall be suppressed at least $70 \mathrm{~dB}$ dealing with the fact that the dynamics of the real input signals varies from $0.3 \mu \mathrm{V}$ to the hundreds of $\mathrm{mV}$.

\section{TECHNICAL SOLUTIONS}

This paragraph compares three different possible architectures of the transceiver and discusses their strengths and weakness. The first and second solution is based on the analog intermediated stage and the last one uses digital design of the receiver and transmitter intermediated stage.

\section{A. Dual Conversion Superheterodyne Receiver}

The dual conversion receiver is a classical architecture of the professional UHF and UHF NFM transceivers. The channel selectivity is realized by a discrete or monolithic quartz filter in the first intermediated frequency. The second intermediate frequency is then used for easier NFM demodulation.

This receiver architecture is featured with an excellent dynamic range and intermodulation products suppression. The main problem is the price of the intermediate filters. In addition, the receiver requires an individual filter or set of filters for each channel spacing.

The NFM modulator is embedded into the carrier frequency synthesizer. The analog voice signal is directly fed to the VCO. The problem is with the modulation of sub-speech signals (tones) that are used for signaling. This modulation cannot be fed to the VCO because of its cancelation by PLL. This sub tones must directly modulate frequency standard. 


\section{B. Direct Conversion Receiver}

Direct conversion receiver to the baseband is a compromised solution that is widely used in semiprofessional transceivers because of its low cost and absence of the expensive intermediate filters that are replaced by baseband ones. The programmable filters can be integrated into the chip. This solution is not currently suitable for professional application because of many technical compromises in dynamic range, intermodulation product suppression and sensitivity.

\section{C. $D U C / D D C$}

The realization of the transceiver by digital intermediate frequency stages $[9,10]$ is a modern trend that brings new quality in the system design, minimized implementation losses that are typical for analog systems. The problem could be higher power consumption in contrast to the analog solution because of using passive filters etc. The critical component of this approach is an $\mathrm{A} / \mathrm{D}$ and $\mathrm{D} / \mathrm{A}$ conversion of the signal that must work in a very wide dynamic range.

The Digital Up Converter (DUC Fig. 1) is the intermediated stage that digitally processes the complex envelope of the modulated frequency and transforms it to the carrier frequency or intermediate frequency in which the digital to analog conversion is realized.

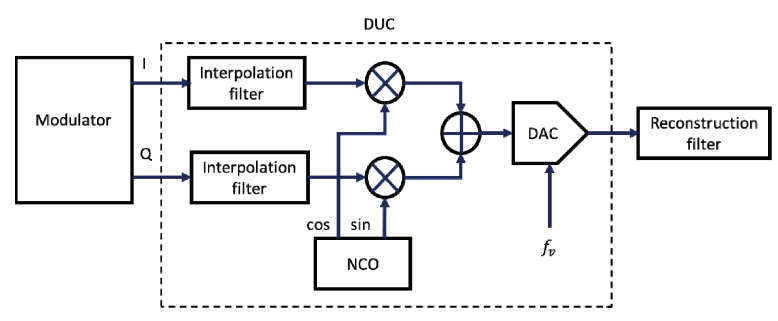

Figure 1. Digital Up Converter

The Digital Down Converter (DDC, Fig. 2) is an digital intermediate stage that processes radio frequency or intermediated frequency signals, realizes channel selectivity, signal decimation, and transformation to the complex envelope for further processing in the demodulator.

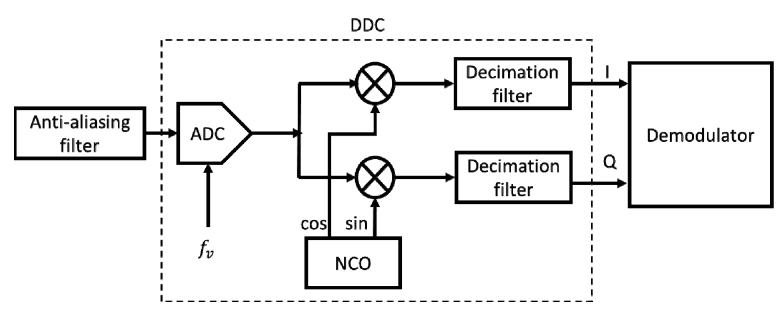

Figure 2. Digital Down Converter

The heterodyne frequencies for digital mixing are generated by Numerical Controlled oscillators that are created by a digital part of Direct Digital Synthesizer and enable precise digital control of the generated signal. Moreover, this approach does not depend on the environment while the classical VCO application in the analog transceivers depends on many factors like ambient temperature, power supply voltage, mechanical stress, etc.

\section{Comparison DUC versus Analog modulator}

The analog frequency modulator is very simple because of the direct modulation of the carrier frequency generator, but there are some weaknesses:

- Variation of the tuning sensitivity of the VCO. The problem is solved by using lower frequency deviation to be able to satisfy standards. The usage of lower deviations causes degradation of the signal to noise ratio during demodulation. This degradation is included in so-called implementation losses.

- Problematic modulation of the low signaling frequency like CTCSS tones in analog mode. This sub-tone modulation is fed directly to the frequency standard as the tones are lower than self-resonant frequency of the frequency synthesizer.

On the other hand, the digital approach based on DDC requires much more complex hardware. The current technology does not allow us to modulate directly at the carrier frequency and comply with strict requirements on the spectral mask. The modulation must be done at intermediate frequency and the resulting product must be up converted to the carrier frequency by an analog up converter. The strengths of this method are an unprecedented quality of modulation signal and excellent manufacture repeatability.

\section{E. DDC versus Superheterodyne and Direct Conversion Receiver}

The superheterodyne is well-mastered technology that enables to design of the high-quality receivers. The professional VHF and UHF receiver hardly makes to achieve this without high quality but expensive quartz filters. The receiver profits from a wide dynamic range and low intermodulation distortion.

The DDC enables to implement a receiver with nearly perfect parameters without linear distortion of the processed signals. The signal processing can be implemented without significant implementation losses in contrast to the "analog" stages. The channel selectivity can be realized by a programmable digital filter. The implementation of the various channel spacing is not a problem. The critical part of the DDC is an ADC, that limits the performance.

\section{ARCHITECTURE OF THE DUC/DDC}

The proposed DUC/DDC is designed for standard intermediate frequency $45 \mathrm{MHz}$. which enables to use relatively cheap but high performance 16 bits ADC for direct sampling of the intermediate signals. In addition, the intermediate frequency is high enough for designing the RF front-end filter with at least $70 \mathrm{~dB}$ suppression of the image frequency.

The digital interface sampling frequency is $96 \mathrm{kSPS}$, i.e. integer multiple of the symbol frequency of the DMR and standard sampling frequency of the audio codecs. The sampling frequency of the ADC is $38 \mathrm{MHz}$ and is programmable to be able to eliminate the spurious response of the receiver caused by a sampling frequency and its harmonic products. The 
sampling frequency of the DAC in DUC is always four times higher.

The intermediate frequency signal of the DDC is down-converted to the baseband by a digital quadratic mixer and then processed by digital decimation filters.

The DUC is designed for processing of the constant envelope signal modulated by any type of analog or digital angular modulation. The up-sampled signal modulates directly the numerically controlled oscillator.

The high decimation and interpolation factor digital filters are based on Cascaded integrator-comb filter (CIC) architecture. The fine filtering of the baseband signals is done by 256 taps FIR filter that is featured with no distortion in the ideal case and very low distortion in a real case.

The DUC/DDC is programmed to the low-cost FPGA that communicates with the host processor by SPI bus.

The functional sample of the DUC/DDC is programmed into the low-cost FPGA kit that is connected by an Arduino connector with a low-cost microcontroller card populated with ARM Cortex M7 microcontroller. The analog to digital and digital to analog converter together with the extremely low jitter sampling frequency synthesizer are integrated to the expansion card, Fig. 3.

\section{TEST RESULtS}

The functional sample of the DUC and DDC (Fig. 3) was thoroughly tested. We measured a dynamic range for the passband signal that is more than $90 \mathrm{~dB}$. The second main tested parameter was DDC selectivity for 25,20 and $12.5 \mathrm{kHz}$ channel spacing. The results are in Fig. 4-6. The stopband shall be attenuated at least $70 \mathrm{~dB}$ which was successfully achieved.

The quality of the modulator was tested on the spectrum of the unmodulated carrier frequency. According to $[1,2]$ the suppression of the side lobes shall be at least $100 \mathrm{dBc}-\mathrm{Hz}$ on frequency offset min $+/-9 \mathrm{kHz}$ or $75 \mathrm{~dB}$ for spectral analyzer resolution bandwidth $300 \mathrm{~Hz}$. It was successfully fulfilled, see Fig. 7.

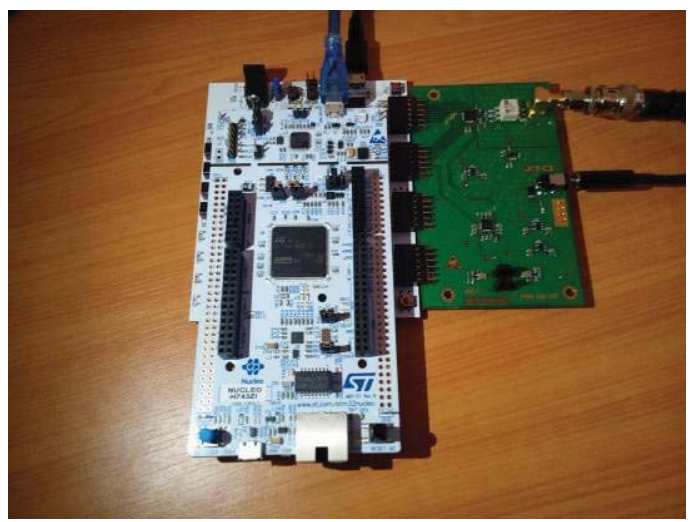

Figure 3. DUC/DDC functional sample

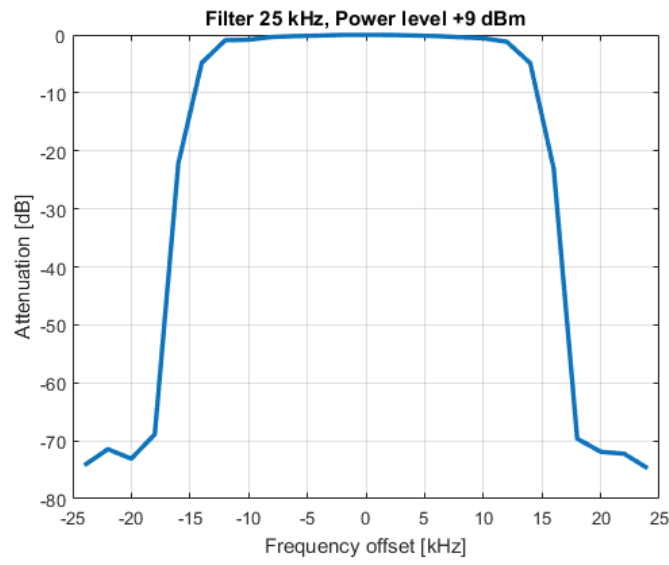

Figure 4. DDC selectivity for $25 \mathrm{kHz}$ channel spacing

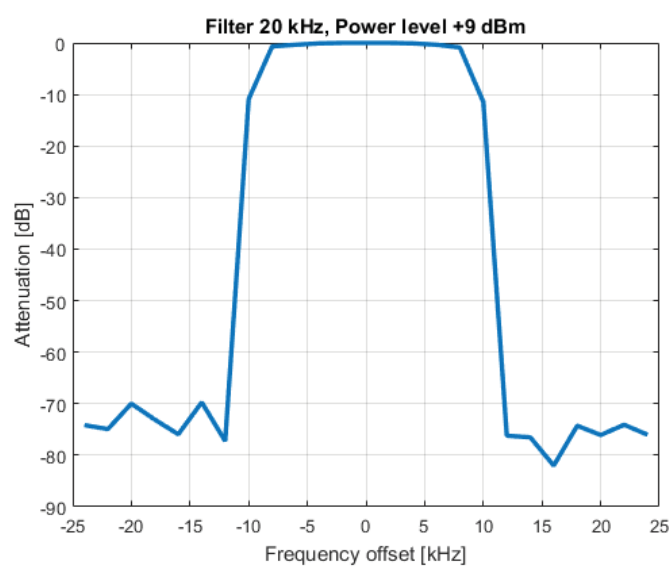

Figure 5. DDC selectivity for $20 \mathrm{kHz}$ channel spacing

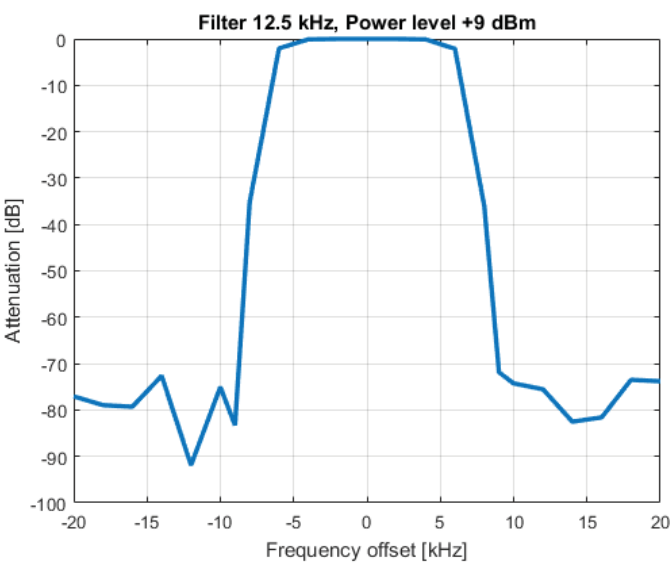

Figure 6. DDC selectivity for $12.5 \mathrm{kHz}$ channel spacing

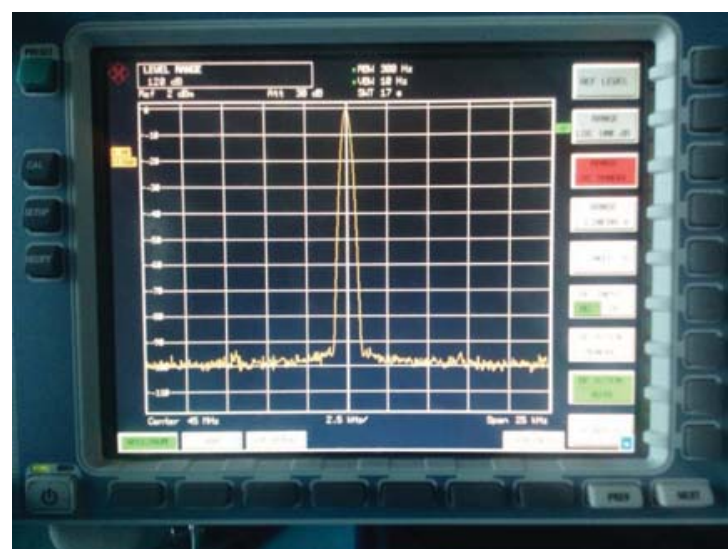

Figure 7. Frequency spectra of unmodulated carrier frequency 


\section{APPLICATION FOR SMALl SATELlite COMMUNICATION}

The communication system of the small research satellite of CubeSat or PocketCube categories is usually based on UHF or VHF low power long-range low-rate low-bandwidth communication because of tight energy budget and limited gain of the satellite antenna. The communication is affected by a huge Doppler shift of the signals caused by a satellite movement. The maximal Doppler shift for various frequencies is in Tab. I.

TABLE I. MAXIMAL DOPPLER SHIFT ON LEO ORBIT

\begin{tabular}{|c|c|}
\hline $\begin{array}{c}\text { Carrier frequency } \\
{[\mathrm{MHz}]}\end{array}$ & $\begin{array}{c}\text { Doppler Shift } \\
{[\mathrm{kHz}]}\end{array}$ \\
\hline 100 & $+/-2.3$ \\
\hline 150 & $+/-3.5$ \\
\hline 400 & $+/-5.2$ \\
\hline 800 & $+/-10.5$ \\
\hline
\end{tabular}

The Doppler frequency, especially on UHF band, is higher than the bandwidth of the modulated signal. The bandwidth of the receiver therefore must be much wider than the bandwidth in the case of the terrestrial communication. The excessively wide bandwidth of the receiver worsens the Bit Error Rate of the typical non-coherent demodulators. The estimated signal losses are approximately from 3 to $5 \mathrm{~dB}$ in UHF band and cause that the maximal bit rate of the data link is less than half in comparison with the optimal case.

This extreme wideband approach is widely used in the design of the small satellite UHF and VHF links. The communication is based on the existing subGigahertz transceiver chips. These chips are widely used in the amateur and semi-professional CubeSats.

The proposed DUC and DDC enables to solve the Doppler problem at the base station without compromises by the software methods well known for example from the SigFox SDR Base station [14].

\section{CONCLUSIONS}

The originality of the paper can be seen in the proof of successful implementation of the narrow band DUC/DDC transceiver for VHF and UHF frequency band. These transceivers are traditionally designed by an analogue way with the usage of the discrete or monolithic quartz filters and double balanced diode mixers because of the very high requirements on the dynamic range and adjacent channel selectivity. On the other hand, DUC/DDC architecture was in the past traditionally applied in the wideband transceivers where the requirements on to the dynamic range and selectivity is not so high. The presented solution provides benefits of both approaches.

The digital processing of the signal in UHF and VHF narrow band transceivers can bring the new quality to the professional transceivers that enables to minimize implementation losses in both receiver and transmitter parts. The signal can be processed with very low linear distortion; the implementation of the various channel spacing is not a problem because of easy reprogramming of the digital filters. The quality of the modulated signal is better than at the transceivers with classical analog angular modulators.

The improvement of the production repeatability and saving of the human work together with the advance of the technical parameters can improve production economy and increase the competitiveness of the transceiver at the market.

\section{ACKNOWLEDGMENT}

This research was sponsored by grant FV30365 "Mobile radio with digital signal processing" of the Ministry of Industry of the Czech Republic.

\section{REFERENCES}

[1] "Land Mobile Service; Radio equipment with an internal or external RF connector intended primarily for analogue speech; Harmonised Standard covering the essential requirements of article 3.2 of the Directive 2014/53/EU" ETSI EN 300086 V2.1.2 (2016-08).

[2] "Land Mobile Service; Radio equipment intended for the transmission of data (and/or speech) using constant or nonconstant envelope modulation and having an antenna connector; Harmonised Standard covering the essential requirements of article 3.2 of the Directive 2014/53/EU", ETSI EN 300113 V2.2.1 (2016-12)

[3] "Electromagnetic compatibility and Radio spectrum Matters (ERM); Digital Mobile Radio (DMR) Systems; Part 1: DMR Air Interface (AI) protocol“, ETSI TS 102 361-1 V1.2.1 (2006-01).

[4] "BENEFITS AND FEATURES OF DMR" White Paper, DMR Association, 2012

[5] "DMR Radio performance characteristics and channel sharing with other radio types", DMR association white paper, http://dmrassociation.org/, March. 2019.

[6] D. C. Cox, "Universal digital portable radio communications," in Proceedings of the IEEE, vol. 75, no. 4, pp. 436-477, April 1987. doi: 10.1109/PROC.1987.13755

[7] Wei Luo, Jie Zeng, Xin Su, Lili Liu and Chen Zhang, "On the implementation of DMR system based on SDR structure," 2013 8th International Conference on Communications and Networking in China (CHINACOM), Guilin, 2013, pp. 314317.

[8] H. Lim, T. Kim, D. Lee and S. Kang, "LARECD: Low area overhead and reliable error correction DMR architecture," 2017 International SoC Design Conference (ISOCC), Seoul, 2017, pp. 27-28. doi: 10.1109/ISOCC.2017.8368812

[9] S. Creaney, I. Kostarnov, "Designing Efficient Digital Up and Down Converters for Narrowband Systems", XAPP1113 (v1.0), Xilinx, November 2008.

[10] "DUC/DDC Compiler v3.0", Xilinx, PG147 November 18, 2015.

[11] A. S. Borozdin and Y. O. Myakochin, "Decoding of DMRStandard Block Codes on the 1967BH034 Processor," 2017 IVth International Conference on Engineering and Telecommunication (EnT), Moscow, 2017, pp. 72-76. doi: 10.1109/ICEnT.2017.22

[12] M. Kang, M. Song and S. Oh, "Design of Transceiver for TETRA in UHF Band," 2007 International Symposium on Microwave, Antenna, Propagation and EMC Technologies for Wireless Communications, Hangzhou, 2007, pp. 1404-1407. doi: 10.1109/MAPE.2007.4393541

[13] L. Noor and Alagan Anpalagan, "Direct conversion receiver for radio communication systems," in IEEE Potentials, vol. 24, no. 5, pp. 32-35, Dec. 2005. doi: 10.1109/MP.2005.1594006

[14] "Sigfox Technical Overview", SigFox White Paper, www.sigfox.com, March 2019 